\title{
Des hommes avec deux chromosomes $X$
}

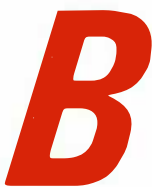
ien que le mécanisme de détermination $d u$ sexe demeure encore totalement incompris, il est communément admis que ce processus est soumis à un contrôle génétique strict chez les mammiferes. C'est ainsi qu'on apprend dès le lycée que le chromosome $Y$ exerce un effet dominant dans la détermination du sexe des gonades.

En l'absence de chromosome $Y$ le sexe est généralement féminin, c'est le cas des caryotypes $45, X$ (syndrome de Turner) et 47, $X X X$, alors qu'en présence d'un chromosome $Y$ le sexe est masculin comme dans le cas des caryotypes $47, X X Y$ (syndrome de Klinefelter) et $47, X Y Y$.

Malheureusement, comme souvent, le dogme souffre un certain nombre d'exceptions connues sous le nom d'inversions du sexe, qui se caractérisent par une discordance entre sexe phénotypique et caryotytique. On connaît ainsi un certain nombre de femmes de caryotype $X Y$, mais la masculinité $X X$ est l'inversion la mieux caractérisée. Les mâles $X X$ apparaissent le plus souvent de fafon sporadique et se détectent en général à l'âge adulte du fait de leur stérilité. Leur taille est inférieure à la normale, mais ils ont une orientation psycho-sociale typiquement masculine et ne manifestent pas de retard mental. Différentes hypothèses ont été avancées pour expliquer ce phénotype. Certaines postulent la présence d'une partie $d u$ chromosome $Y$, de taille insuffisante pour pouvoir être décelée dans un caryotype, alors que d'autres sont basées sur l'existence d'une mutation sur $X$ ou un autosome. Ces hypothèses sont longtemps restées sans réponse satisfaisante.

Depuis quelques années, à l'instar de nombreux autres domaines de la biologie, la technologie de l' $A D N$ recombinant a permis de faire avancer le $\mathrm{m} / \mathrm{s} n^{\circ} 6$ octobre 85 problème de fafon considérable. Dans un travail conjoint avec le groupe de Marc Fellous à l'Institut Pasteur, nous avons pu montrer, voici 2 ans, la présence de fragments d'ADN caractéristiques $d u$ chromosome $Y$ humain dans le génome de mâles $X X[I]$, présence mise à jour par hybridation à des sondes moléculaires spécifiques. Plus récemment, par des études moléculaires systématiques portant sur un grand nombre de cas sporadiques de masculinité $X X$, nous avons pu établir la présence d'un segment contigu (d'un seul tenant) $d u$ chromosome $Y$ humain dans au moins $60 \%$ des cas examinés (cas $Y+$ ). Il est plus que vraisemblable que le phénotype masculin présenté par les mâles $X X Y+$ est une conséquence de la présence d'ADN spécifique du chromosome $Y$.

La partie du chromosome présente chez les mâles $X X$ est variable selon les individus. Toutefois, la partie présente chez les individus ayant refu le segment le plus petit est toujours présente chez les individus ayant hérité d'un segment chromosomique de plus grande taille. Ce phénomène de recouvrement inclusif $d u$ plus petit segment par le plus grand suggère un transfert orienté de matériel chromosomique et concorde avec une mobilisation incluant une des régions terminales (télomère) du chromosome $Y$. Des expériences de cartographie plus précise ont permis d'assigner le segment transféré, au bras court du chromosome $Y$. Il est d'ailleurs possible d'ordonner les différents fragments d'ADN détectés sur une structure linéaire et d'utiliser l'étude des mâles XX comme méthode d'établissement d'une carte de fragments d'ADN du chromosome $Y$ humain. $E n$ raison de son état normalement haploïde, le chromosome $Y$ reste le seul qui n'ait pu être représenté par une carte quelque peu informative et la méthode mentionnée ci-dessus constitue en fait la première tentative sérieuse de cartographie des différentes régions du chromosome $Y$ humain. Les sondes isolées à ce jour ne recouvrant pas la totalité du chromosome $Y$, il reste impossible de formuler une conclusion définitive concernant les cas de masculinité $X X$ apparemment $Y-$. Il est par ailleurs apparu récemment et de fafon inattendue que certains cas familiaux étaient également $Y+[2]$. Des résultats similaires avaient également montré que la mutation "sex-reversal ", responsable chez la souris d'un phénomène héréditaire de masculinité $X X$, a pour origine une translocation d'un segment du chromosome $Y$ murin sur le chromosome $X[3]$. Un modèle analogue est généralement avancé pour expliquer la masculinité $X X$ sporadique chez l'homme. Cette hypothèse est actuellement examinée dans divers laboratoires par des expériences d'hybridation in situ sur des chromosomes, à l'aide des sondes $Y$-spécifiques que nous avons isolées à l'Institut Pasteur.

La compréhension d'un phénomène paradoxal permet souvent de mieux cerner les questions essentielles. $A$ ce titre, l'étude de la masculinité $X X$ a permis d'ouvrir une nouvelle voie d'approche dans le problème de la détermination du sexe.

J. W.

I. Guellaën $G$, Casanova $M$, Bishop $C$, et al Human $X X$ males with $Y$ single-copy $D N A$ fragments. Nature 1984; 307: 172-3. 2. Page $D C$, de la Chapelle $A$, Weissenbach 7. Chromosome $Y$-specific DNA in related human $X X$ males. Nature 1985; 315: 224-6.

3. Singh $L$, Jones $K W$. Sex reversal in the mouse (Mus musculus) is caused by a recurrent nonreciprocal crossover involving the $X$ and an aberrant $Y$ chromosome. Cell 1982; 28: 205-16. 